\title{
Polarisation in Political Twitter Conversations
}

\author{
David Gunnarsson Lorentzen, Swedish School of Library and Information Science, Borås, Sweden
}

The author would like to thank the anonymous reviewers for their valuable and insightful comments.

\section{Introduction}

Being exposed to various topics and different points of view is central to democracy, but new technologies run the risk of making its users more prone to fragmentation and polarisation, which could in turn lead to difficulties in understanding non-like-minded and extremism, as well as distorted understanding of issues (Sunstein, 2009). However, Sunstein also suggests that when many different groups are participating in conversations, society will be exposed to their different opinions and might be better off as a result. Compared to other information tools on the web, Twitter allows the user greater control of information sources. Whereas search tools such as Google mobilises techniques to personalise content based on usage history, preferences and, in many cases, friends' search history, on Twitter the information is better controlled by the user, who can choose which actors and hashtags to follow. Given these allowances, Twitter is an interesting application to study when investigating issues of polarisation. This article is focused on how different groups with separate political views connect to each other on Twitter during non-election times. Will polarisation be visible despite the allowances of Twitter or is this an interest-based social network that successfully connects people beyond their political comfort zone?

Twitter's potential of facilitating these kinds of discussions has been questioned. So far a number of studies have come to the conclusion that politicians' activity on Twitter largely follows one-way communication patterns (e.g. Sæbø, 2011; Grusell \& Nord, 2012). Sæbø also noted that most tweets do not support the deliberative model of democracy. However, Bruns and Highfield (2013, p. 671) claimed that Twitter can provide "a simple mechanism for citizens to invoke politicians [...] in their comments, and for these thoughts to be public and visible in a way that emailed communication, telephone calls, letters, or electorate office visits are not".

Only a few studies have explored the interaction between different groups within a political conversation. These have analysed interaction between pro-life and pro-choice advocates (Yardi \& boyd, 2010), networks of re-tweets and mentions (Conover et al., 2011), overlapping of sets of followers of politicians (Kim \& Park, 2012), mentions of politicians (Bruns \& Highfield, 2013), and replies and re-tweets by politicians (Paßmann et al., 2014). A mention is the use of another Twitter actor's username in a tweet, which also has the function of a directed message. This paper aims to investigate not only the mentions, but also the re-tweets and the followership relations. Relying on networks based on messages when studying communication has drawbacks. First, it only captures active actors and not actors that have important network positions but are less active. Second, any undirected message is directed to, or intended for, all the actor's followers, or actors following any hashtag included in the tweet. Therefore, this paper explores alternative methods, using social network analysis of followership among the actors, and their communication within the hashtagged set of tweets. The analysis is focused on the most prominent actors of Swedish political conversations, regardless of whether the actors are politicians or not. The research questions are:

- To what extent do members of the different political groups relate to and communicate with members of other groups?

- How do the sub-networks comprised by these groups compare to each other with regards to connectedness, looking at intra-group and inter-group relationships and communication?

- How do the followership, mentions, and re-tweets networks compare with regards to polarisation? 
At the time of this study there were two political blocks in the parliament; the left block, and the centre-right block. One party in the parliament was not member of any block. This party was the Sweden Democrats (social conservatives with a nationalistic ethos). The centre-right block consisted of the Moderate Party (liberal conservatives), the Liberal Party (liberals), the Centre Party (green, decentralised liberals), and the Christian Democrats (Christian democrats). The left block consisted of the Social Democratic Party (social democrats), the Green Party (green ideologists), and the Left Party (socialists). Of the 349 representatives in the parliament, 173 were from the centre-right parties and 156 from the left parties (The Swedish Parliament, 2013).

Political activity on the web by the Swedish parties has been studied before. Larsson's (2011) longitudinal study of party web sites revealed that, in general, the parties were better at informing than involving, mobilising, and connecting, with connecting being their overall weakest point. Centre-right parties were better than the others at informing but worse at involving. In mobilising, parties from the left and centre-right blocks were amongst those with the highest as well as the lowest scores. The Sweden Democrats were amongst the worst performers in all categories, most notably in connecting.

Twitter activity and attitudes towards Twitter as communication channel was studied by Grusell and Nord (2012). They found that most of the activity was focused on informing rather than interaction, with the Liberal Party, the Centre Party and the Green Party having higher interaction levels, and the Sweden Democrats having no interaction at all.

\section{Literature review}

Since the application was launched in 2006, there have been a number of studies of its usage and content. Relevant for this paper are those concerning political usage. These have focused on predicting election results (e.g. Metaxas et al., 2011), tweeting during election campaigns (e.g. Larsson \& Moe, 2012; Bruns \& Highfield, 2013), predicting political preferences and affiliations (e.g. Pennacchiotti \& Popescu, 2011), examining the correlation between political opinion polls and sentiment words (e.g. O'Connor et al., 2010), and usage by political leaders or governmental organisations (e.g. Lassen \& Brown, 2011). This section highlights studies that are relevant for this paper, i.e. such that are hashtag and network based studies of political Twitter usage. The section concludes with a number of studies of polarisation on Twitter.

One major topic of interest has been the conversations in conjunction with elections. Bruns and Burgess (2011) described key patterns of activity as well as thematic foci during the 2010 Australian election by following the hashtag \#ausvotes. They found that activity was often event-related and peaked during election day. A similar finding was made by Larsson and Moe (2012) in their study of the Swedish 2010 election. They tracked the hashtag \#val2010, and did a social network analysis based on mentions and re-tweets of and by the most active actors. This group was dominated by politicians, journalists and bloggers. At state level, political activity and campaigning was studied by Bruns and Highfield (2013) as they followed members of the parliament and alternative candidates during the 2012 state election in Queensland, Australia. Apart from this, they also tracked \#qldvotes and compared the activity around the candidates with the overall hashtagged activity. Among their findings was the conclusion that ordinary Twitter users are mentioning the politicians to talk about them rather than for conversational purpose.

Some studies have focused on more general political conversations on Twitter. Small (2011) applied content analysis to tweets containing the Canadian political hashtag \#cdnpoli. Mass media actors contributed to about $10 \%$ of the tweets, whereas politicians accounted for less than $2 \%$. Tweets were focused mainly on informing, but very few tweets could be identified as reporting of news. The Austrian political discussions were studied by Ausserhofer and Maireder (2013), with a focus on relations between political actors and citizens. It was concluded that the Austrian political Twittersphere was dominated by an elite of political professionals, but also that the discussions were open to participating citizens. 
Few examples exist regarding exploration of polarisation or homophily in political conversations on Twitter. Yardi and boyd (2010) studied interaction in abortion conversations. Their analysis was based on tweets with replies where senders and repliers were coded into one of the two categories pro-life and pro-choice. The results indicated that while Twitter users were more likely to communicate with like-minded, there were also examples of heterogeneity. Conover et al. (2011) examined political polarisation during the 2010 US congressional midterm elections by analysing re-tweet and mention networks, and found that the former revealed a polarised structure, but also that the latter did not indicate polarisation. It was concluded that users might try to provoke an interaction by injecting partisan content into the opposing actors' timelines. A study of re-tweets and replies by politicians was performed in a Dutch context by Paßmann et al. (2014). The findings indicated that politicians mainly re-tweeted members of their own party, but replied to members of different parties. The aforementioned study of Bruns and Highfields (2013) also included an analysis of cross-boundary communication, showing that Twitter actors mention other actors from not just one, but several different political groupings. It was also shown that cross-boundary mentioning existed between the party organisations. A final example is Kim and Park's (2012) study of followers of five Korean politicians from four different parties, which found little overlaps between the sets of followers, suggesting that polarisation exists in the chosen context.

\section{Method}

The following section describes and discusses issues of how to access Twitter data, the algorithm for identifying the most prominent actors, the analysis of profile descriptions, and the analysis of networks.

\subsection{Data collection}

For gathering Twitter data, an adapted version of yourTwapperKeeper was used. Data were collected by tracking the hashtag \#svpol ("Swedish politics") during four weeks in September 2012. This hashtag was first identified in a pilot study by tracking the hashtag \#almedalen during the Almedalen Week, which is a political event. \#svpol was, apart from the tracked hashtag, the far most frequently used hashtag. It was chosen as it is used for general political discussion. All other frequently used Swedish political hashtags are used for specific issues (e.g. \#migpol for "migration politics") or specific events (e.g. \#almedalen). Tracking a hashtag is "a manageable and low-cost alternative" (Bruns \& Stieglitz, 2013, p. 92). The goal is not to capture the entire conversation, but rather a sample of it. The main focus here is to map the relationships and the communication among the participating actors. According to Bruns (2011), studies of mention patterns within a set of tweets containing a hashtag can identify the most central users within the topical network of the hashtag. This paper extends the method by also including followership and retweet networks.

In the dataset used for this paper 10,294 actors were present. To make a manual categorisation of actors possible, a subset containing the top tweeters was created algorithmically each of the four weeks by calculating an actor score for the actors that had contributed with at least five tweets during the week. Drawing on Anger and Kittl (2011), the score was calculated from the activity and the visibility of each actor, as well as its probability of spreading a message throughout the network. A weighting scheme was used as basis for the calculations. Larger weights were given to original tweets and those tweets that were being re-tweeted, as well as to those tweets that addressed other actors (Table 1). The intended focus of the paper is on the users that at some stage contributed sufficiently to the conversations by their activity, but also the users that the community signaled as prominent by its activity. This method captures those with a relative contribution to the conversations, as well as different types of actors, including those who are frequent tweeters, and those who are frequently mentioned or re-tweeted. A drawback on this method is the neglecting of qualitative aspects of the tweets and the activity.

[insert Table 1 about here]

The list of actors was sorted by the average value, and the top 500 were kept. The four lists were then aggregated into one final list of 916 actors, labelled "prominent actors" in this paper. Previous studies have shown that political 
conversations on Twitter are dominated by a small number of users (e.g. Jürgens et al., 2011; Bruns \& Highfield, 2013), and this study is no different with the prominent actors accounting for $72 \%$ of all tweets.

The profile data, which include a description of the actor written by the actor itself, were collected alongside the lists of friends, by calling the Twitter API's users/show and friends/ids for each of these actors. For 17 actors, an error message was returned and no profile or friends data could be retrieved, possibly due to the account being suspended or removed. Hence, these 17 actors were excluded from the network analyses. All the Twitter IDs were re-coded for ethical reasons so that no political views could be associated with a physical person.

There are some issues that have implications on the result. boyd et al. (2010) found that there is no agreed-upon syntax for a re-tweet, but that the prototype is "RT @ user Text". A problem is that Twitter's re-tweet button embeds the tweet in the actor's timeline without adding RT and the original tweeter's username (Bruns, 2011). The compromise solution was to follow the prototype "RT @user Text", accepting that some re-tweets cannot be identified. It should also be noted that not all replies are hashtagged, so the network of mentions underestimates replies (Bruns \& Stieglitz, 2013). With the lack of replies, the mention analysis is merely an analysis of initiatives to conversations (Bruns, 2011), rather than of full conversations.

\subsection{Analysing data}

The profile descriptions were analysed manually. Following the groupings in the parliament, the actors were categorised into the left block (L; 136 actors), the centre-right block (CR; 116), the right-wing block (RW; 62), and other blocks (23), based on the content in their profile descriptions. It was also noted if the account belonged to an active politician (professional or non-professional), a political party, or a mass media actor. In total, 189 political accounts were found. 71 accounts were related to mass media actors, four of these considered being political. The networks reveal a large number of actors categorised as "Other", including 67 mass media actors. This is a methodological constraint following the focus on actors that are open about their identities. The approach could be complemented by analysis of the tweets. However, profile data were in this paper chosen over tweet content because it is more consistent over time, arguably more reliable, and less open to arbitrary interpretation.

Social network analysis was utilised to map the relationships and the communication between actors. In social networks, nodes (the actors) are connected by edges (the relationship between the nodes). Of interest here is the followership and communication within and between the groups. While the focus of the paper is to analyse the relationships across the block boundaries, knowledge of relationships within the blocks is valuable to contextualise the inter-block findings. For example, are actors more willing to follow and communicate with like-minded than non-like-minded, and if so, to what extent? Different types of visualisations of networks were created using the Gephi software (Bastian et al., 2009), and laid out using the ForceAtlas2 algorithm. The ForceAtlas 2 algorithm does not take attributes of nodes into account, as it depends only on the connections, and the weight of the edges (Jacomy et al., 2012). Thus the algorithm emphasises the tighter connected clusters in the networks, which is suitable for the purposes of this paper. As edge weight is taken into consideration, the actors that interact more frequently are closer to each other in the weighted graphs (mentions and re-tweets). The networks, with disconnected nodes excluded, are:

1. Followership network (893 nodes, 48,401 edges). The edge is drawn from the follower to the followed actor.

2. Mentions network (693 nodes, 3,995 edges). The edge is drawn from the actor that is mentioning to the mentioned actor.

3. Re-tweets network ( 851 nodes, 6,417 edges). The edge is drawn from the re-tweeting actor to the actor who posted the original tweet.

Two network metrics are used in this paper. These are density and degree centrality, where density is an indicator of connectedness of a network, given as the number of connections in a graph divided by the maximum number of connections, and degree centrality is an indicator of how central a node is, given as the number of connections the 
node has with other nodes (Wasserman \& Faust, 1994, pp. 129, 178). This paper uses two versions of degree centrality: normal and weighted, where the latter takes the number of interactions as a weight.

Finally, the actors were divided into groups based on their activity and visibility (see Bruns \& Stieglitz, 2013). The groups follow a 90/9/1 division where the $1 \%$ most active and visible actors respectively formed one group. The activity was also broken down into these actions:

- Original tweets: original statements not including mentions,

- Mentions: mentions of other actors including messages directed to other actors but excluding re-tweets,

- Re-tweets, and

- Tweets containing URLs.

\section{Results and discussion}

The nodes in the network graphs are sized according to their degree centrality value. In the mentions and re-tweets graphs, the weighted degree is used.

\section{INSERT FIGURE 1 HERE}

\subsection{Group level analysis}

The followership graph (Figure 1; left) reveals that the members of the three main groups are clustered together. More than $95 \%$ of the actors within these followed fellow group members. At the bottom left, the RW group is somewhat distant to the other groups, even though there are some CR actors situated close to or even within the RW cluster. There are more connections between the clusters of CR actors (right of the graph) and L actors (top), and as a consequence these clusters are closer in the graph. The network analysis shows that the actors follow accounts related to other groups to some extent (Table 2). The average CR actor followed and was followed by $7 \mathrm{~L}$ actors. The average $\mathrm{L}$ actor followed and was followed by $6 \mathrm{CR}$ actors. There is very little connection between $\mathrm{L}$ and $\mathrm{RW}$. Actors from all three groups followed an equal number of accounts from other groupings (41 friends), but the rightwingers had more followers per actor (49; CR: 42; L: 37). Overall, RW had slightly more friends (65; CR: 63; L: 62), and more followers per actor than the other two (72; CR: 64; L: 57).

[insert Table 2 about here]

The groups differed from each other regarding intra-group followership. RW was by far the densest network with a density of 0.38 (Table 3). All networks had a similar average degree, but as the RW group was smaller, these connections were also distributed over fewer actors. CR and L had a similar density value, with the former slightly denser than the latter. All three networks were denser than the network as a whole.

Contrary to the followership graph, the mentions graph (Figure 1; centre) is sparser and different in its structure as there are no signs of polarisation. Actors seem to address the like-minded actors as well as others to the same extent, which is also confirmed by Table 2. Overall, RW actors sent significantly more messages than members of the other groups. RW was also the only of these three groups that sent more messages than it received. L actors were the least active in sending messages. CR and L had a reciprocal communication, with $73 \mathrm{CR}$ messages sent to $\mathrm{L}$ and 75 messages sent in the opposite direction. The communication between CR and RW was also fairly reciprocal, with 69 messages from CR and 62 from RW. However, RW sent 111 messages to L, but only received 19. The intra-group activity differed among the groups. RW actors communicated significantly more with each other than with the other groups. The RW network is much denser than the other two and has a significantly higher average weighted degree (Table 3).

The re-tweets graph (Figure 1; right) is similar to the followership graph, but sparser. There is a significant difference in the messaging behaviour compared with the re-tweet behaviour. Table 2 shows the total number of re- 
tweets made by the groups in the "Out" columns and the total number of re-tweets of the own tweets in the "In" columns. For all main groups, a large share of the re-tweets made by group members were re-tweets of messages posted by other group members. RW actors were the far most frequent re-tweeters with a total of 4,427 re-tweets (L: 1,606; CR: 976). RW actors also had more tweets re-tweeted (2,922; L: 1,275; CR: 1,216). Re-tweeting tweets from one of the other two main groups was generally not as common as mentions, even though RW actors re-tweeted CR messages 267 times.

[insert Table 3 about here]
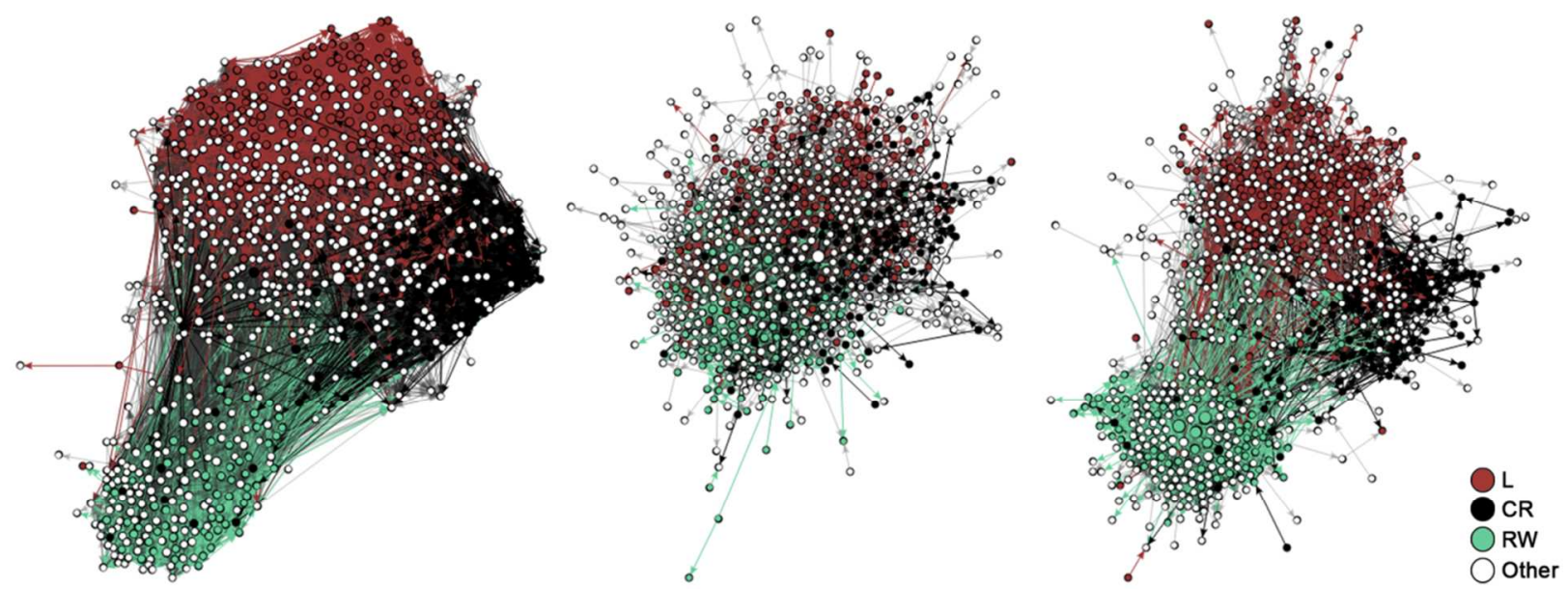

Figure 1. Network graphs. Left: followership, centre: mentions, right: re-tweets.

Table 4 shows the differences between the networks. The mention network suggests that actors seem to be as willing to communicate with members of other blocks as with members of their own block. All groups had a mention ratio close to 1, which gives little support for polarisation. RW is the exception when comparing followership and retweets network. Its members re-tweeted tweets from other blocks to a larger extent and as a group it was the only one with a followership ratio close to the re-tweet ratio. Overall though, the re-tweet network is more polarised than the followership network. As the hashtagged set of tweets does not contain replies without the hashtag, the mention network cannot fully function as an indicator of polarisation.

[insert Table 4 about here]

\subsection{General statistics}

Of the 77,436 collected tweets, $45 \%$ were original tweets, $18 \%$ included mentions, and $37 \%$ were re-tweets. $51 \%$ contained a URL. This can be compared to previous results by Larsson and Moe (2012), who found 60.2\% original tweets, 32.8\% re-tweets and 7\% directed messages, and Small (2011), who found that $7 \%$ of the tweets were mentions and commented re-tweets.

The analysis of the activity by user percentile groups (Figure 2) shows similar results as Bruns and Highfield (2013). The least active $90 \%$ did not contribute as much as the other groups. When they did, a larger percentage of their tweets were re-tweets. The share of mentions was larger in the top 1\% group, and the top 10\% had a larger share of original tweets. The use of URLs was very similar across all groups, even though the least active group had a marginally larger share of tweets including URLs than the other groups. A difference between these results and those of Bruns and Highfield is that a larger share is comprised by original tweets in this set, at the expense of mentions. 
When comparing the three main political groups (Figure 3), a different pattern emerges. Although all groups posted a similar amount of original tweets, and, except for the left block, a similar amount of mentions, the right-wingers posted far more re-tweets. $49.6 \%$ of their tweets were re-tweets, which is quite similar to the least active $90 \%$ group.

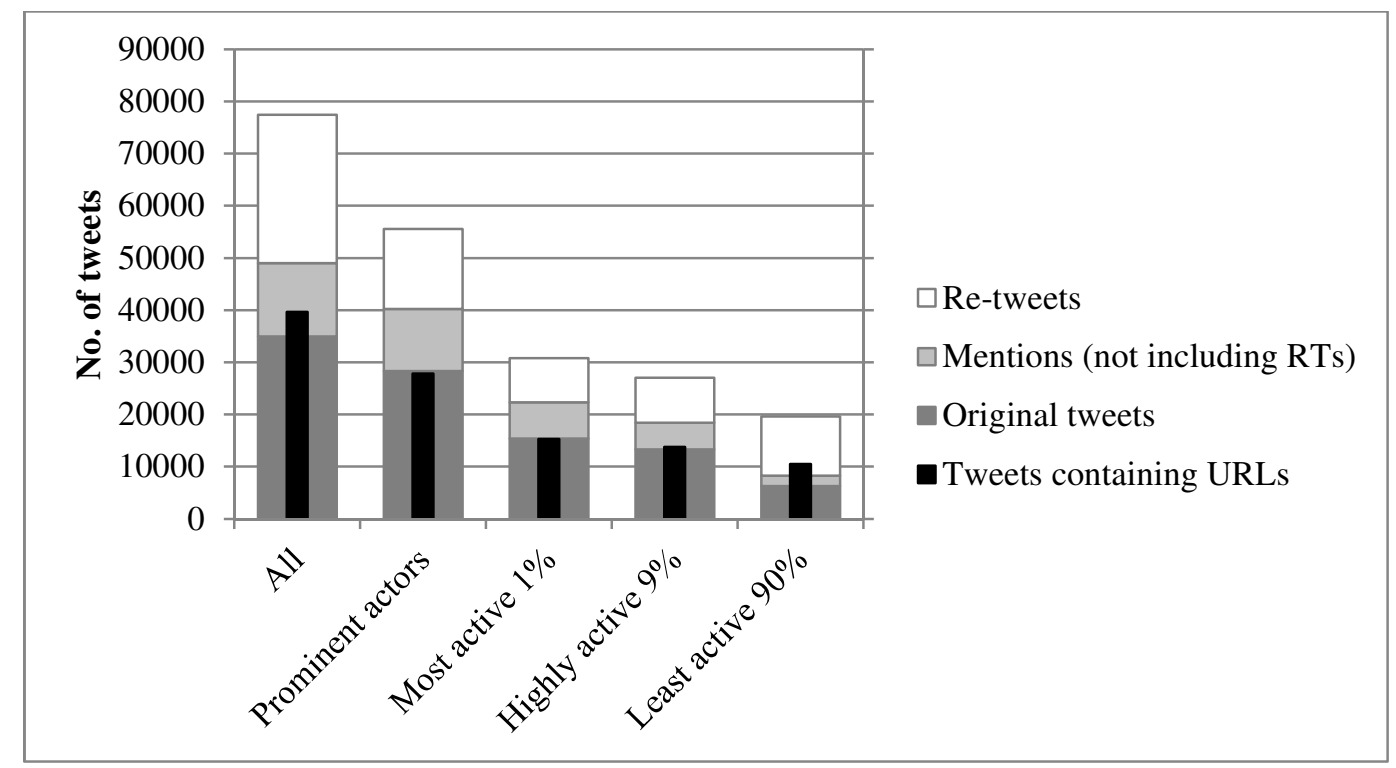

Figure 2. Activity by actor percentiles, compared to the whole group of actors, and the group of prominent actors.

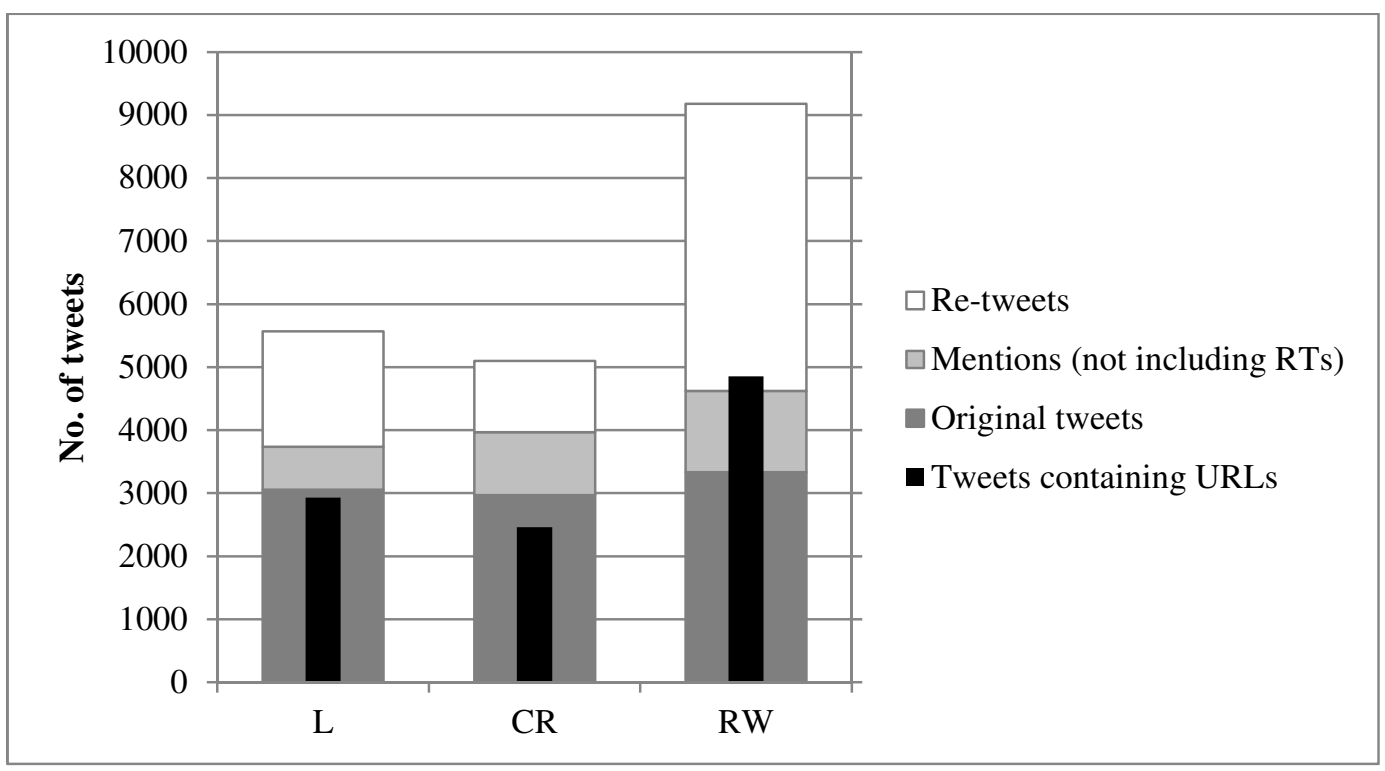

Figure 3. Activity by main political groups.

This study found little support for claims that Twitter activity is dominated by elite users such as journalists and politicians. Only 11 mass media actors and 7 political actors were in the top percentile for activity. For visibility, the corresponding figures were 8 and 15. It seems as during non-election times, the conversations are dominated by ordinary users.

\section{Conclusions and directions for future research}


This paper analysed communication and relationships across political boundaries based on three different kinds of networks. The analysis revealed clear examples of polarisation, but also that people are using the allowances of Twitter to create connections across boundaries. Polarisation is evident in the re-tweet and followership networks, but not in the mention network. However, if replies were included in the dataset, the latter network would perhaps be clustered differently.

The RW group was most different from the others, with a much higher density in all three networks. Its actors were more outreaching, as well as they attracted more followers per actor. The right-wingers were also the most frequent re-tweeters with a re-tweet share only comparable to the least active $90 \%$. This is in contrast to the findings of the two studies of Swedish parties' usage of the web (Larsson, 2011) and Twitter (Grusell \& Nord, 2012). The numerically strong representation, and the high activity, of right-wingers is an interesting finding, considering the potential of Twitter to function as a means of outreach for minority actors (Larsson \& Moe, 2012). For the historically strong actors in politics, keeping an eye on the activity of a hashtag like the one used here could be a good strategy to keep track on these actors.

The L and CR blocks have a stronger representation in the parliament, which they also have had historically, and a strong standing in the traditional media. This might entail a more restrictive usage of social media. Given their tradition of competing and discussing with each other, it is not surprising that they have a reciprocal communication, and that their relational inter-connectedness is stronger. But these blocks also differ from each other, with CR actors more often mentioning other actors, and L actors least active in initiating conversations, but more often re-tweeting. Given that both relationships and communication is different within sub-groups of the conversations, research needs to look at the whole as well as the parts of the conversations. It will be interesting in further research to see if these kinds of sub-groups, with their different characteristics and the levels of cross-boundary followership and communication, can be found in different contexts (e.g. different countries, in general versus event related conversations).

Overall, the results indicate that Twitter actors do interact across boundaries, but that they prefer to follow and retweet like-minded actors. The latter point is important as it follows that the actors are less likely to be exposed to for them controversial opinions. However, followers of the hashtag are likely to be exposed to a diverse range of opinions, given that actors with different political affiliations are active users of the hashtag. This is a unique affordance of Twitter which could enable depolarisation. If following a hashtag means that actors are exposed to different kinds of opinions from like-minded as well as non-like-minded, it would be very interesting to analyse the replies. Who replies to whom? What is the nature of the replies to a like-minded compared to a non-like-minded? Assuming that Twitter's search API retrieves a large enough portion of the streamed tweets, and that no actors or tweets are systematically excluded by Twitter, the streaming API could be utilised to track the users in the search set, to collect tweets to them that are in reply to any of the hashtagged tweets. Future research should aim to include replies to tweets in hashtag studies.

There are some other points that need to be addressed qualitatively. First, RW actors sent far more messages to L than vice versa. As these groups are supposed to be farther from each other than other groups in this setting it would be tempting to follow up on Conover et al. (2011) and investigate if actors are trying to provoke interaction with actors from other groupings. It could also be an effect of a minor actor trying to be included, with traditionally strong actors trying to keep the distance. Moreover, Twitter is arguably a tool more suitable for individuals rather than parties, and this analysis found a significant number of accounts belonging to individual politicians. It is possible that Twitter's affordances could shift the focus of the conversations from the parties to the individual politicians and their personal opinions. For these reasons, the nature of the tweets would be an interesting subject to investigate. Are the mentions used as, for example, substitutes for politicians' names as suggested by Bruns and Highfield (2013) or are they truly conversational, and how do politicians communicate in relation to their parties? 
Second, RW actors re-tweeted actors from the other groups more often than vice versa. Given that they have more followers per actor, they help other groups to spread their messages. But what is the type of messages re-tweeted, who are the actors that re-tweet, and who are they followed by? A re-tweet could be an indication of agreement of opinions in specific issues, but the possibilities and habits of modifying messages when re-tweeting (boyd et al., 2010) also makes it possible to attribute content to someone who is not responsible for it, or to modify the content that someone was responsible for. This entails that the audience viewing a re-tweet may be notified of a conversation they might be interested in, but they may also get the wrong idea of an actor's opinion should the message have been altered. Therefore, cross-boundary re-tweets need to be analysed qualitatively in order to find out why an actor from one group is re-tweeting an actor from another group. These points have implications for politicians. Just counting mentions and re-tweets is not good enough to measure success on Twitter. Being aware of what happens to the tweets when they are re-tweeted by competitors and of how the members of the party are addressed, is important.

The combination of followership and communication data enable us to find actors that are well connected through followership but not active in their communication, and actors that are active in their communication but not well connected. This was not investigated further in this paper, but for future studies, it could be interesting to find what kind of actors that are well connected but less active, and vice versa. A final question to pose is whether findings regarding elite actors such as journalists and politicians are results of what is analysed (e.g. activity/visibility or degree in conversation networks), when the conversations take place (e.g. election or non-election times), or type of hashtag (e.g. general or event related)? A recommendation for future research is to combine analysis at the group level with analysis of the individuals, and also analyse their activity and visibility relative to the conversations as a whole. 


\section{References}

Anger, I., and Kittl, C. (2011), “Measuring Influence on Twitter”, in Lindstaedt, S., and Granitzer, M. (Eds.), Proceedings of the 11th International Conference on Knowledge Management and Knowledge Technologies, ACM, New York.

Ausserhofer, J., and Maireder, A. (2013), "National politics on Twitter", Information, Communication \& Society, Vol. 16 No. 3, pp. 291-314.

Bastian M., Heymann S., and Jacomy M. (2009), “Gephi: an open source software for exploring and manipulating networks", in International AAAI Conference on Weblogs and Social Media, pp. 361-362.

boyd, d., Golder, S., and Lotan, G. (2010), “Tweet, tweet, retweet: conversational aspects of retweeting on Twitter”, in Proceedings of the 2010 43rd Hawaii International Conference on System Sciences (HICSS '10), IEEE Computer Society, Washington, pp. 1-10.

Bruns, A. (2011), "How long is a tweet? Mapping dynamic conversation networks on Twitter using Gawk and Gephi”, Information, Communication \& Society, Vol. 15 No. 9, pp. 1323-1351.

Bruns, A., and Burgess, J. (2011), “\#ausvotes: How Twitter Covered the 2010 Australian Federal Election”, Communication, Politics \& Culture, Vol. 44 No. 2, pp. 37-56.

Bruns, A., and Highfield, T. (2013), "Political networks on Twitter", Information, Communication \& Society, Vol. 16 No. 5, pp. 667-691.

Bruns, A., and Stieglitz, S. (2013), “Towards more systematic Twitter analysis: metrics for tweeting activities”, International Journal of Social Research Methodology, Vol. 16 No. 2, pp. 91-108.

Conover, M. D., Ratkiewicz, J., Francisco, M., Goncalves, B., Flammini, A., and Menczer, F. (2011), "Political polarization on Twitter", in Proceedings of the 5th international conference on weblogs and social media, pp. 89-96.

Grusell, M., \& Nord, L. (2012), "Three Attitudes to 140 Characters: The Use and Views of Twitter in Political Party Communications in Sweden”, Public Communication Review, Vol. 2, No. 2, pp. 48-61.

Jacomy, M., Heymann, S., Venturini, T., and Bastian, M. (2012), “A graph layout algorithm for handy network visualization”, working paper, Médialab, Sciences Po, Paris, 1 August, available at http://www.medialab.sciencespo.fr/publications/Jacomy_Heymann_Venturini-Force_Atlas2.pdf (accessed 18 December 2013)

Jürgens, P., Jungherr, A., and Schoen, H. (2011), "Small Worlds with a Difference: New Gatekeepers and the Filtering of Political Information on Twitter", in Proceedings of the 3rd International Web Science Conference, June 14-17, 2011, ACM, New York.

Kim, M., and Park, H. W. (2012), "Measuring Twitter-based political participation and deliberation in the South Korean context by using social network and Triple Helix indicators”, Scientometrics, Vol. 90, No. 1, pp. 121-140.

Larsson, A. O. (2011). “'Extended infomercials' or 'Politics 2.0’? A study of Swedish political party Web sites before, during and after the 2010 election”, First Monday, Vol. 16, No. 4.

Larsson, A. O., and Moe, H. (2012), "Studying political microblogging: Twitter users in the 2010 Swedish election campaign”, New Media \& Society, Vol. 14, No. 5, pp. 729-747.

Lassen, D. S., and Brown, A. R. (2011), “Twitter: The electoral connection?”, Social Science Computer Review, Vol. 29 No. 4, pp. 419-436. 
Metaxas, P. T., Mustafaraj, E., and Gayo-Avello, D. (2011), "How (Not) To Predict Elections", in Proceedings of the IEEE International Conference on Privacy, Security, Risk, and Trust, and International Conference on Social Computing, pp. 165-171.

O’Connor, B., Balasubramanyan, R., Routledge, B. R., and Smith, N. A. (2010), "From Tweets to Polls: Linking Text Sentiment to Public Opinion Time Series", in Proceedings of the International AAAI Conference on Weblogs and Social Media, Washington, DC, The AAAI Press, pp. 122-129.

Paßmann, J., Boeschoten, T., and Schäfer, M. T. (2014), "The gift of the gab. Retweet cartels and gift economies on Twitter”, in Weller, K., Bruns, A., Burgess, J., Mahrt, M., and Puschmann, C. (Eds.), Twitter and Society, Peter Lang, New York, pp. 331-344.

Pennacchiotti, M., and Popescu, A.-M. (2011), "Democrats, Republicans and Starbucks Afficionados: User Classification in Twitter", In Proceedings of the 17th ACM SIGKDD international conference on Knowledge discovery and data mining (KDD '11), ACM, New York, pp. 430-438.

Small, T. A. (2011), "What the hashtag? A content analysis of Canadian politics on Twitter", Information, Communication \& Society, Vol. 14 No. 6, pp. 872-895.

Sunstein, C. R. (2009), Republic.com 2.0, Princeton, N.J.: Princeton University Press.

Sæbø, Ø. (2011), “Understanding Twitter ${ }^{\mathrm{TM}}$ Use among Parliament Representatives: A Genre Analysis”, in Tambouris, E., Macintosh, A., and de Bruijn, H. (Eds.), ePart 2011, LNCS 6847, Springer, Heidelberg, pp. 1-12.

The Swedish Parliament (2013), "Members and parties”, available at http://www.riksdagen.se/en/Members-andparties/ (accessed 18 December 2013).

Wasserman, S., and Faust, K. (1994), Social network analysis: methods and applications, Cambridge: Cambridge University Press.

Yardi, S., and boyd, d. (2010), "Dynamic Debates: An Analysis of Group Polarization Over Time on Twitter", Bulletin of Science Technology Society, Vol. 30 No. 5, pp. 316-327. 


\begin{tabular}{|c|c|c|c|}
\hline Aspect & Metric & Description & Weight \\
\hline \multirow{4}{*}{ Activity } & Original tweets & The number of tweets that are not re-tweets or mentions & 2 \\
\hline & Directed tweets & The number of mentions & 1.5 \\
\hline & Re-tweets & The number of re-tweets posted & 1 \\
\hline & $\begin{array}{l}\text { Outbound } \\
\text { conversation }\end{array}$ & The number of actors mentioned by the actor & 1 \\
\hline \multirow[t]{2}{*}{ Visibility } & Received tweets & The number of tweets received & 1 \\
\hline & Inbound conversation & The number of actors mentioning the actor & 1 \\
\hline \multirow[t]{2}{*}{ Spreadability } & Tweets re-tweeted & The number of own tweets re-tweeted by other actors & 2 \\
\hline & Actors re-tweeting & $\begin{array}{l}\text { The number of actors that have re-tweeted a tweet posted } \\
\text { by the actor }\end{array}$ & 1 \\
\hline
\end{tabular}

Table 1. Metrics for the calculation of the actor score. 


\begin{tabular}{|c|c|c|c|c|c|c|c|c|c|c|c|}
\hline & $\begin{array}{l}\text { Direction } \\
\text { Group }\end{array}$ & $\begin{array}{l}\text { Out } \\
\text { L }\end{array}$ & In & $\begin{array}{l}\text { Out } \\
\text { CR }\end{array}$ & In & $\begin{array}{l}\text { Out } \\
\text { RW }\end{array}$ & In & $\begin{array}{l}\text { Out } \\
\text { All oth }\end{array}$ & $\begin{array}{l}\text { In } \\
\text { er groups }\end{array}$ & $\begin{array}{l}\text { Out } \\
\text { Total }\end{array}$ & In \\
\hline \multirow{3}{*}{$\begin{array}{l}\text { Followership } \\
\text { (avg per } \\
\text { actor) }\end{array}$} & $\mathbf{L}$ & 20.5 & & 5.9 & 5.8 & 0.4 & \multirow{3}{*}{$\begin{array}{l}0.6 \\
2.3\end{array}$} & 41.2 & 36.9 & 61.7 & 57.3 \\
\hline & CR & 6.8 & 6.9 & 21.9 & & 2.1 & & 41 & 42.3 & 62.9 & 64.2 \\
\hline & RW & 1.4 & 1 & 4.2 & 3.9 & 22.3 & & 41.7 & 48.6 & 64.7 & 71.6 \\
\hline \multirow[t]{3}{*}{ Mentions } & $\mathbf{L}$ & 94 & & 75 & 73 & 19 & \multirow{3}{*}{$\begin{array}{l}111 \\
62\end{array}$} & 282 & 876 & 376 & 970 \\
\hline & CR & 73 & 75 & 176 & & 69 & & 497 & 841 & 673 & 1,017 \\
\hline & $\mathbf{R W}$ & 111 & 19 & 62 & 69 & 172 & & 701 & 793 & 873 & 965 \\
\hline \multirow[t]{3}{*}{ Re-tweets } & $\mathbf{L}$ & 626 & & 65 & 39 & 22 & \multirow{3}{*}{$\begin{array}{l}34 \\
267\end{array}$} & 980 & 649 & 1,606 & 1,275 \\
\hline & CR & 39 & 65 & 401 & & 48 & & 575 & 815 & 976 & 1,216 \\
\hline & RW & 34 & 22 & 267 & 48 & 1,443 & & 2,984 & 1,479 & 4,427 & 2,922 \\
\hline
\end{tabular}

Table 2. Cross-boundary relations and communication. 


\begin{tabular}{lllllll}
\hline & Followership & \multicolumn{2}{l}{ Mentions } & \multicolumn{2}{l}{ Re-tweets } \\
& Density & Avg degree & Density & Avg w. degree & Density & Avg w. degree \\
\hline L & 0.15 & 20.49 & 0.004 & 0.69 & 0.018 & 4.6 \\
CR & 0.19 & 21.9 & 0.006 & 1.52 & 0.018 & 3.46 \\
RW & 0.38 & 22.28 & 0.029 & 2.77 & 0.096 & 23.29 \\
Overall & 0.06 & 52.84 & 0.005 & 8.57 & 0.008 & 4.92 \\
\hline
\end{tabular}

Table 3. General network statistics. 


\begin{tabular}{llll}
\hline Group & Followership ratio & Mention ratio & Re-tweet ratio \\
\hline L & 3.23 & 1 & 7.19 \\
CR & 2.46 & 1.24 & 4.61 \\
RW & 4.09 & 0.99 & 4.79
\end{tabular}

Table 4. Comparison between networks. The ratio is own group/the other two groups. 